\title{
HOMEOPATIA NO TRATAMENTO DA ANSIEDADE
}

\author{
HOMEOPATHY IN THE TREATMENT OF ANXIETY
}

\author{
Cláudia Raquel Zamberlam ${ }^{1}$ \\ Adauto Luiz dos Santos ${ }^{2}$
}

RESUMO: A ansiedade é frequentemente relatada por pacientes que buscam tratamentos alternativos ou complementares, como a Homeopatia, por exemplo. A matéria médica compreende grande variedade de medicamentos homeopáticos e evidenciou o uso em pessoas sadias. Contudo, os estudos clínicos carecem de informações sobre os efeitos alcançados. $\mathrm{O}$ extrato da planta Ginkgo biloba mostrou efeitos ansiolíticos em animais roedores, assim como Gelsemium sempervirens, Ignatia amara, Achilea milefolium e outras substâncias de origem vegetal. A busca pelo medicamento individual é um diferencial do tratamento homeopático, no intuito de encontrar o Simillimum ideal para o paciente que sofre ao seu modo determinado sintoma. Este projeto se propõe a levantar evidências sobre o uso da Homeopatia no tratamento de transtornos de ansiedade por meio de casos clínicos no intuito de contribuir para o uso de medicamentos homeopáticos na melhora da saúde dos indivíduos.

Palavras-chave: Homeopatia; Ansiedade; Simillimum; Medicamentos dinamizados; Transtornos emocionais.

ABSTRACT: Anxiety is often reported by patients seeking alternative or complementary treatments, such as Homeopathy, for example. The medical field comprises a wide variety of homeopathic medicines and has shown their use in healthy people. However, clinical studies lack information on the effects achieved. Ginkgo biloba plant extract showed anxiolytic effects on rodent animals, as did Gelsemium sempervirens, Ignatia amara and other substances of plant origin. The search for individual medication is a differential of homeopathic treatment in order to find the ideal Simillimum for the patient who suffers in his particular way. This project aims to raise evidence on the use of homeopathy in the treatment of anxiety disorders through clinical cases to contribute to the use of homeopathic medicines in improving the health of individuals.

Keywords: Homeopathy; Anxiety; Simillimum; Dynamized drugs; Emotional disorders.

\footnotetext{
1 Farmacêutica. Mestre e Doutora em Neurofarmacologia (UNIFESP). Especialista em Homeopatia (UNIATLÂNTICO). Docente do curso de Farmácia da Universidade UNG e Pesquisadora em Fitoterapia e Homeopatia. Instituição: UNG-Universidade Guarulhos. email: crzamberlam@gmail.com.

2 Farmacêutico Clínico, Mestre em Homeopatia (FACIS), Especialista em Fitoterapia e Acupuntura (FACIS), Coordenador do Ambulatório Farmácia Clínica em Homeopatia e Fitoterapia (FACIS). Instituição: Faculdade de Ciências da Saúde-FACIS. E-mail: fitoterapiaadauto@gmail.com.
} 


\section{INTRODUÇÃO}

A ansiedade é um distúrbio que afeta milhões de pessoas em todo o mundo, em todas as faixas etárias, e quando se instala no indivíduo de forma patológica, limita suas atividades diárias laborais e pode levar até mesmo a quadros mais complexos como a depressão. Atualmente, a população está envelhecendo e com isto o estudo de tratamentos para os transtornos cognitivos estão ganhando grande importância no cenário da pesquisa clínica (Fernandes et al, 2017).

Os transtornos comportamentais e ansiedade possuem alta prevalência na sociedade moderna, sendo responsáveis pelo afastamento de trabalhadores de seus ofícios e por produzir gastos de recursos financeiros públicos e privados significativos. Os transtornos de ansiedade são caracterizados por um excessivo e persistente sentimento de medo na ausência de um perigo iminente, ou pela antecipação do mesmo e até mesmo produzindo tensão e desconforto pelo desconhecido (Menezes et al., 2017).

Os medicamentos frequentemente utilizados no tratamento da ansiedade são os ansiolíticos, que na sua grande maioria produzem efeitos depressores do Sistema Nervoso Central (SNC). Os ansiolíticos mais conhecidos são os benzodiazepínicos, que modulam os receptores GABAérgicos, produzindo um efeito agonista, ou seja, semelhante ao neurotransmissor GABA (ácido-gama-amino-butírico), um representante é o Diazepam. A sua ação é inespecífica, ou seja, não possui seletividade para um determinado receptor e por isto ocasiona muitos efeitos colaterais, entre eles, o comprometimento cognitivo e desenvolvimento de dependência e tolerância (Andreatini et al., 200I).

Existem outros tipos de ansiolíticos também conhecidos, por exemplo, a Buspirona e outras drogas pertencentes à classe dos compostos azaspironas que possuem ação mais seletiva, direcionada aos receptores serotonérgicos $\left(5-\mathrm{HT}_{\mathrm{IA}}\right)$. Mesmo sendo mais seletivos, esta classe de medicamentos também possui desvantagens, como por exemplo o comprometimento psicomotor, desenvolvimento de dependência e tolerância ou interação com outras drogas de ação central (Graef, 2002; Andreatini et al., 200I).

É importante considerar que há pacientes ansiosos que não respondem suficientemente ao tratamento com os fármacos clássicos. O uso prolongado de benzodiazepínicos provoca o desenvolvimento tolerância, alterações cognitivas e de memória, dependência física e abstinência na descontinuação do uso (Zulian, 2013).

Neste sentido, a busca por medicamentos naturais que possuem eficácia comparável aos os sintéticos, apresentando diminuição dos efeitos colaterais, é uma valiosa contribuição às opções 
existentes no mercado, para o tratamento de transtornos relacionados à ansiedade (Graef, 20or; Zamberlam et al., 2016).

A Homeopatia é uma especialidade médica e farmacêutica, criada por Samuel Hahnemann, que testou os medicamentos homeopáticos em pessoas sadias em doses infinitesimais, utilizando de medicamentos informacionais e na sua grande maioria não ponderais, no intuito de reduzir ou anular efeitos relacionados à intoxicação, assim caracterizados como medicamentos ultra-diluídos.

Um medicamento dinamizado pode ser obtido a partir de substâncias de natureza mineral, animal ou vegetal, sendo que para cada origem, o método adequado de acordo com a solubilidade da substância ponto de partida. Quando solúvel, obtém-se uma solução hidroalcoólica, quando insolúvel, utiliza-se a técnica da trituração com lactose antes da diluição. A escala centesimal é obtida pela diluição de uma parte da substância ponto de partida diluída em 99 partes de uma solução inerte e após isto, este sistema é agitado por Ioo vezes, assim obtendose a primeira centesimal Hahnemann $(\mathrm{ICH})$. Este processo de diluição e agitação denomina-se dinamização, e a quantidade de dinamizações (diluição e agitação) realizada é o que determina a "potência" do medicamento homeopático.

Evidências mostraram que em ${ }_{12} \mathrm{CH}$, não há presença da substância original. pois atinge concentrações inferiores a 1o 24 Molar, portanto as preparações homeopáticas superiores a $\mathrm{IICH}$ ficam abaixo do número de Avogadro e acima de ${ }_{\mathrm{II}} \mathrm{CH}$ e analogamente, as preparações abaixo desta potência, apresentam certa materialidade (Zacharias, 2002).

A fitoterapia utiliza plantas medicinais com fins terapêuticos. Os estudos baseados em fitoterápicos são úteis no entendimento dos efeitos das drogas vegetais e sustentam o uso de medicamentos dinamizados em potências baixas, em geral, $\mathrm{ICH}$ a $7 \mathrm{CH}$, quando se deseja o efeito farmacológico da planta de origem (Alain Hiorvilleur, 2003).

Estudos de Cerutti, Zamberlam e colaboradores, evidenciaram eu um extrato padronizado de Ginkgo biloba em dose mediana é capaz de promover efeitos ansiolíticos em animais roedores portadores de transtornos de medo condicionado. Contudo, os mecanismos de ação pelos quais o fitoterápico exerce os efeitos ainda não são conhecidos completamente. Sabese que podem ocorrer efeitos tóxicos dependendo da dose e do esquema farmacoterapêutico (Zamberlam et al., 2016).

Assim, o estudo de medicamentos homeopáticos para o tratamento da ansiedade, provenientes de extratos de plantas como Ginkgo biloba ou de outros vegetais, ou ainda de outras 
origens, pode contribuir de forma significativa para a prática clínica (Zamberlam et al., 2or6; Eleutério et al., 2011; Grundmann et al., 2007).

A maneira prática de se diferenciar ansiedade normal de ansiedade patológica é basicamente avaliar se a reação ansiosa é de curta duração, autolimitada e relacionada ao estímulo do momento ou não. Quando a ansiedade ou o medo são exagerados, são caracterizados como patológicos e interferem com a qualidade de vida, o conforto emocional ou o desempenho diário do indivíduo e podem ser assim reconhecidas como Transtornos de Ansiedade Generalizada (TAG), apresentando, em geral, um curso crônico, que na sua maioria devem ser tratados com abordagem multidisciplinar como psicoterapia e terapia farmacológica (Castillo et al., 20oo; Fontes, 2013).

Este trabalho se desenvolve com abordagem sobre a Homeopatia, seus conceitos, história e períodos importantes. São apresentados conceitos sobre os transtornos de humor, bem como são relatados os trabalhos de pesquisa com medicamentos homeopáticos para o tratamento de emoções como medo, ansiedade e depressão, assim como trabalhos desenvolvidos em pesquisa básica, em animas ou em outros protocolos de estudo.

\section{OBJETIVOS}

\section{I Objetivo geral}

Este estudo se propõe a contribuir com a literatura sobre os benefícios da terapêutica homeopática, evidenciando os benefícios da Homeopatia no transtorno de ansiedade.

\subsection{Objetivos específicos}

- Apresentar evidências do uso de medicamentos dinamizados no tratamento de transtornos da ansiedade.

- Proporcionar esclarecimentos sobre os efeitos da medicina naturista.

- Contribuir com a comunidade na divulgação dos efeitos benéficos que a Homeopatia oferece para o tratamento de transtornos emocionais.

\section{JUSTIFICATIVA}

Os estudos relacionados ao uso de medicamentos dinamizados no tratamento de transtornos de ansiedade são escassos e permanecem não claros e parte dos artigos encontrados são revisões bibliográficas (Eleutério et al., 20II). 
No entanto, existem alguns trabalhos que contribuem com maiores informações sobre os efeitos de medicamentos ultra diluídos na melhora de parâmetros considerados ansiolíticos em modelos animais, entre as matérias médicas estudas podemos encontrar Gelsemium sempervirens, Ginkgo biloba, Ignatia amara, entre outras (Bellavite et al., 2018; Zamberlam et al., 2016; Yang et al., 2007; Filho et al., 2010).

- Este trabalho se propõe a realizar uma busca por dados acerca do uso de medicamentos homeopáticos no tratamento de transtornos de humor, contribuindo com as evidências dos benefícios da Homeopatia no tratamento da ansiedade. Entre os casos apresentados neste estudo, enumeram-se:

- Prática homeopática na ansiedade e depressão em cuidados primários;

- Homeopatia na depressão em estudo randomizado, parcialmente duplo-cego, placebocontrolado;

- Uso da Ignatia amara em modelos comportamentais em animais;

- Efeitos do Gelsemium sempervirens na ansiedade e neurologia experimental;

- Análise dos efeitos de Ginkgo biloba em fitoterapia, em modelo de resposta emocional em roedores, como base para o uso de potências baixas na Homeopatia;

- Ensaios experimentais sobre o uso de medicamentos homeopáticos no comportamento de roedores e modelos psicopatológicos.

\section{HIPÓTESE}

A hipótese deste trabalho é que a ansiedade pode ser melhorada mediante o tratamento homeopático.

\section{METODOLOGIA}

Este trabalho se desenvolveu por meio de revisão de literatura, com a finalidade de reunir o conhecimento científico já publicado sobre o assunto, o que permite analisar criticamente as evidências disponíveis em bases de dados nacionais, revistas publicadas por pesquisadores e indexadores de produção científica.

Como critérios de inclusão, foram selecionados artigos publicados na língua inglesa ou portuguesa, com abordagem geral sobre tratamento homeopático em transtornos de ansiedade. Contudo, devido à escassez de estudos, foram incluídos no estudo artigos e publicações sobre Homeopatia nos transtornos de humor tais como depressão ou medo, por meio da plataforma BIREME- Centro Latino-Americano e do Caribe de Informação em Ciências da Saúde, com 
acesso às bases de dados na área da saúde: Literatura Latino Americana e do Caribe em Ciências da Saúde (LILACS), e Biblioteca Eletrônica Científica Online (SciELO).

Foram utilizados os descritores: "Homeopatia"; "Ansiedade"; "Simillimum"; "Medicamentos dinamizados"; "Transtornos emocionais". publicados, preferencialmente nos últimos vinte (20) anos até 2020. Esta janela cronológica foi determinada considerando a disponibilidade de trabalhos publicados na área. Foram excluídos do estudo as publicações que não apresentavam informações relacionadas ao tema proposto ou correlacionados.

\section{FUNDAMENTAÇÃO TEÓRICA}

\section{I A História da Homeopatia}

A atividade médica era apoiada no conhecimento experimental, sistematizado pelo pai da medicina, Hipócrates (468 a 377 a.C), que acreditava que a doença era uma perturbação no equilíbrio entre o ser humano e a natureza. Assim, ele demonstrou que as doenças eram resultantes da reação naturais de defesa do organismo frente ao meio ambiente. Hipócrates estabeleceu determinantes até hoje utilizadas na prática da medicina que são o diagnóstico, prognóstico e a terapêutica (Brasil, 2019, Pustiglione, 2019; Mamtani 2002).

O pensamento de Hipócrates influenciou no nascimento da medicina homeopática, que se baseou no pensamento hipocrático: "a doença é produzida pelos semelhantes e pelos semelhantes o paciente retorna à saúde”, o que se conhece como o princípio da similitude: Simília similibus curantur, que significa, o semelhante é curado pelo seu semelhante. Esta linha de pensamento não estava de acordo com as ideias adotadas na época, que pregavam a cura pelos contrários, ou seja. Contraria contrariis curantur, como é a filosofia da alopatia tradicional, que utiliza substâncias que atuam contrariamente aos sintomas das doenças (Robinson, 2006; Colin, 2000).

A Homeopatia está fundamentada na observação experimental que a substância que seja capaz de provocar determinados sintomas em um indivíduo, é também capaz de curar um outro doente que apresente os mesmos sintomas, em doses adequadas e não tóxicas (Shamples et al., 2003).

Christian Friedrich Samuel Hahnemann (1755 - 1843) foi responsável pelo surgimento da Homeopatia em um contexto de grande agitação na Europa durante a Revolução Francesa. Este contexto foi marcante, pois trazia as ideias de libertação que se espalhavam pelo mundo (Corrêa et al., 2006; Pustiglione et al., 2017). 
Nascido em Meissen, cidade pequena localizada na Saxônia, Alemanha, em 1755. Durante a sua vida morou em várias cidades. Em 1779 defendeu a sua tese de doutorado na Universidade de Erlangen-Nuremberg, Alemanha, tornando-se doutor em medicina, exercendo esta profissão por muitas cidades germânicas. Durante a época da sua atuação como médico, percorreu muitas cidades e conheceu muitas pessoas, inclusive Lavoisier e outros influentes pensadores e homens da ciência da época (Sanchez, 2013).

Em 1789 abandona a medicina e passa a dedicar-se à pesquisa. Ao traduzir a bibliografia médica de Dr. Cullen, leu sobre o efeito da quinina e decidiu testar em si mesmo, captando a relação entre a doença natural e a doença artificial induzida pela substância. Publicou em 1796 o trabalho: "Experimentação Pura" e continua reunindo dados para a Matéria Médica (Corrêa et al., 2006; Pustiglione et al., 2017).

Tanto trabalho e a sua avançada idade desgastou a sua saúde, quando falece em 2 de julho de I843, aos 88 anos, sendo sepultado em Montmartre e posteriormente os seus restos mortais foram exumados e transferidos para Paris (Sharples et al., 2003; Pustiglione, 1998).

\subsection{Avanços científicos da Homeopatia}

A Organização Mundial da Saúde (OMS) tem apoiado a inclusão das Práticas Integrativas Complementares (PICs) de tratamentos na atenção à saúde das pessoas, e a Homeopatia está inserida nestas práticas. O objetivo comum desta inclusão é ajudar a salvar vidas e proporcionar melhores condições de saúde, estimulando a investigação clínica sobre segurança e eficácia dos tratamentos complementares e para facilitar o intercâmbio de informac,ões (Zullian, 2013; Brasil, 2006.).

Pustiglione, em 2017, ao realizar um breve panorama sobre a prática de Homeopatia como especialidade médica, refere que as PICs não devem se chocar com a medicina convencional e sim complementar-se em harmonia benéfica, e salienta que a experiência tem mostrado que a integração de ambas é útil na promoção da saúde em doenças agudas e crônicas (Pustiglione, 2017).

Estima-se que cerca de 500 milhões de pessoas fazem uso da terapêutica homeopática, representando uma parcela considerável da população mundial e acarreta a necessidade do desenvolvimento de ensino desta ciência. Na União Europeia, 6 dos 22 países possuem regulamentação da Homeopatia no sistema de saúde (WHO, 2017).

No Brasil, no final do século XIX, a Homeopatia foi reconhecida como prática médica e farmacêutica, através do decreto imperial n.ำ 9.554 de 3 de fevereiro de 1886, o qual oficializava as farmácias homeopáticas. $\mathrm{Na}$ época, a prática médica predominante eram a alopatia e a cirurgia. 
O primeiro livro impresso foi publicado por Pedro Ernesto Albuquerque de Oliveira em São Paulo em 1886: "Da Febre Typhoide e Enfermidades Sobrevientes no Brasil e o seu Tratamento Homeopático". A partir de então, outras especialidades médicas como os médicos veterinários, cirurgiões dentistas, farmacêuticos passaram a fazer parte da prática homeopática (Brasil, 2019, Pustiglione, 2019).

Além disto, observou-se o impacto da chegada da Homeopatia no Brasil por meio de menções em obras literárias como "A moreninha" de Joaquim Manuel de Macedo (I820 - I882) e em relatos pessoais de José Bento Monteiro Lobato (1882 - 1948) numa carta a seu amigo Moura Rangel na qual comenta sobre o uso de medicamentos homeopáticos como Mercurius e Sulphur no seu filho (Pustiglione, 2019).

Em 2018, a medicina homeopática completou roo anos de reconhecimento no Brasil, pelo decreto legislativo n. ${ }^{\circ} 3.540$ de 25 de setembro de 1918. O ensino de farmacotécnica homeopática tornou-se obrigatório nos cursos de Farmácia em 1952 através da Lei n.o․ 1.552 (Corrêa et al., 2006).

No Brasil, a Homeopatia foi incluída entre as Práticas Integrativas e Complementares através da portaria n. ${ }^{\circ}$ 971 do Ministério da Saúde, em 2006, através da Política Nacional de Práticas Integrativas e Complementares (PNPIC) do Sistema Único de Saúde -SUS, por entender que a Homeopatia possui potencial de prevenção, promoção e recuperação da saúde.

Considerando o crescimento das PICs na área da saúde e, além disto, tendo em vista a necessidade de contribuir com o corpo de publicações científicas sobre a Homeopatia, este estudo se propõe a realizar uma revisão de literatura sobre o uso de medicamentos homeopáticos em indivíduos com transtornos de ansiedade.

\subsection{Homeopatia nos transtornos de ansiedade}

No Brasil, a prevalência de transtornos de ansiedade é de $9,3 \%$ da população, de acordo com dados da OMS. Estes números se destacam no cenário mundial, que é de 3,6\%, já na América os transtornos ansiosos atingem 5,6\% da população. Acredita-se que a dinâmica agitada da vida moderna contribui para essas estatísticas, muitas vezes ocasionando problemas no convívio social e diminuição do rendimento profissional. Além disto, os transtornos de ansiedade não devidamente tratados, tendem a se tornarem crônicos com o tempo, exigindo maior durabilidade do afastamento laboral (Who, 2017; Batelaan et al,2014).

Um estudo transversal analisou a prevalência de transtornos de ansiedade que ocasionaram afastamentos laborais no município de Teresina, Piauí em 2017 onde foi observado 
que a maior incidência ocorreu em mulheres de 20 anos com afastamento de $4 \mathrm{I}$ dias em média (Fernandes et al, 2018).

Apesar de ser considerada uma reação normal, de adaptação e necessária ao organismo, considerando que é uma resposta contra ameaças ou perigos, a ansiedade pode se tornar uma patologia, quando o indivíduo perde a autopercepção e o equilíbrio frente às reações fisiológicas e psicológicas das reações em relação a uma determinada situação, exacerbando-as desproporcionalmente e levando à evitação ou fuga (Schmidt et al., 20II, Moraes et al., 2015).

A Homeopatia aplica o princípio da cura pela similitude (cura pelos semelhantes) pelo uso de substâncias previamente experimentadas em indivíduos sadios em doses infinitesimais. Utiliza matéria-prima dos reinos animal, mineral e vegetal em busca do equilíbrio orgânico. Segundo Eleutério, 20II, a prática da homeopatia pode ser aplicada como coadjuvante em procedimentos odontológicos, principalmente em crianças que apresentam transtornos de ansiedade e medo (Eleutério et al., 20II).

Em um estudo clínico foi demonstrado que a Homeopatia pode tratar a ansiedade do paciente nos momentos que antecedem um atendimento odontológico. Foi realizada uma comparação do tratamento convencional com o homeopático em pacientes que apresentavam medo de dentista e que se autodeclaravam ansiosos frente aos procedimentos odontológicos.

Um estudo evidenciou que o tratamento homeopático resultou em maior taxa de redução da ansiedade após 90 dias de tratamento, comparado com os ansiolíticos convencionais, além de não resultar em efeitos colaterais, o que ocorreu com os medicamentos alopáticos tradicionalmente usados para o controle da ansiedade. No estudo foram analisados grupos tratados com Diazepam ou com medicamento homeopático e foi encontrado maior redução da ansiedade no grupo tratado com medicamento homeopático e além disto, foi relatado um alto percentual de efeitos adversos no grupo tratado com Diazepam, enquanto não foi observado nenhum efeito adverso no grupo que recebeu Homeopatia (GIORGI, 2010).

Ainda, Bousta evidenciou o efeito ansiolítico de Gelsemium sempervirens na potência $5 \mathrm{CH}$ em camundongos testados na tarefa comportamental de presença no compartimento claro/escuro, que mensura o nível de ansiedade do animal (Bousta et al, 20or).

Magnani e colaboradores em 2010 realizaram uma pesquisa com camundongos com o objetivo de analisar efeitos de G. sempervirens na ansiedade. Foram testadas cinco diluições centesimais Hahnemannianas: ${ }_{4} \mathrm{CH},{ }_{5} \mathrm{CH},{ }_{7} \mathrm{CH},{ }_{9} \mathrm{CH}$ e $\left.30 \mathrm{CH}\right)$ e comparadas com um medicamento de uso comum na alopatia, a Buspirona. Os animais foram testados nas tarefas de Campo Aberto (CA) e Caixa de Claro-Escuro (CCE). G. sempervirens aumentou a porcentagem 
de tempo gasto no compartimento da luz, um indicador de atividade do tipo ansiolítica, com um efeito estatisticamente significativo usando as diluições ${ }_{5} \mathrm{CH},{ }_{9} \mathrm{CH}$ e $30 \mathrm{CH}$. Esses efeitos foram comparáveis aos da buspirona. O número de transições entre os compartimentos do teste CCE aumentou acentuadamente com as diluições de G. sempervirens ${ }_{5} \mathrm{CH},{ }_{9} \mathrm{CH}$ e $30 \mathrm{CH}$. Eles concluíram que o padrão geral de resultados fornece evidências de que G. sempervirens atua na reatividade emocional de camundongos (Magnani et al., 2010).

Os estudos de Magnani foram contestados por Cervo \& Torri, que publicaram comentários em 2010 sobre os efeitos ansiolíticos da preparação homeopática de Gelsemium sempervirens, no qual questionaram possíveis erros experimentais, entre eles, na interpretação dos comportamentos dos animais e nas análises estatísticas. Eles acreditam que outros metabólitos presentes na planta possam também contribuir para os resultados, além da Gelsemina, para qual se atribui os efeitos ansiolíticos encontrados, no entanto, não é considerado que o medicamento é ultra diluído, e que os efeitos encontrados não se relacionam às doses ponderais e sim à informação medicamentosa deixada pela matéria-prima no veículo dinamizado (Cervo \& Torri, 2012; Liu et al., 2013).

Entre os mecanismos que medeiam a ansiedade, pode-se citar diferentes sistemas de neurotransmissão, entre eles o GABAérgico, o serotonérgico e ainda dopaminérgico, bem como considera-se a participação de neuropeptídeos (Graef 20oI).

Pesquisas realizadas com extratos de plantas medicinais também têm sido realizadas em modelos animais. Baretta e colaboradores, em 2012, avaliaram o efeito do extrato hidroalcoólico de partes aéreas de Achillea millefolium L. em camundongos submetidos ao teste de ansiedade denominado Labirinto em Cruz Elevado (LCE) e no teste de Campo Aberto. Ambos avaliam o nível de ansiedade por meio da observação comportamental dos animais. Foi demonstrado que o extrato da planta atua como ansiolítico comparado aos medicamentos para controle da ansiedade tradicionais, e além disto, propõem um mecanismo de ação diferente dos receptores GABA (Ácido Gama Amino Butírico), classicamente envolvidos com os ansiolíticos da classe dos benzodiazepínicos, utilizados na alopatia (Baretta et al., 2012).

Estes achados representam uma alternativa para o tratamento da ansiedade, no sentido de que propõe uma estratégia de prevenção de desenvolvimento de dependência e tolerância de benzodiazepínicos (DZP). Conforme visto por Ennaceur, em 2010, o fármaco Diazepam, da classe dos DZP provoca o desenvolvimento de sensibilização e tolerância em camundongos testados na tarefa da plataforma elevada, além de evidenciar o efeito "dependente de estado" da droga, o que 
sugere que o medicamento não induz à cura da ansiedade no indivíduo, e sim, promove apenas o efeito enquanto o sujeito está sob efeito da substância (Ennaceur \& Michalikova, 2010).

Os tratamentos farmacológicos utilizados para o tratamento dos transtornos da ansiedade incluem as classes de medicamentos benzodiazepínicos e antidepressivos. Um estudo randomizado, duplo-cego, controlado por placebo, dose flexível, comparou os medicamentos antidepressivos cloridrato de trazodona e cloridrato de imipramina com o ansiolítico benzodiazepínico diazepam e foi relatada uma melhora acentuada em $73 \%$ dos pacientes tratados com imipramina, 69\% dos pacientes tratados com trazodona, $66 \%$ dos pacientes tratados com diazepam, mas apenas $47 \%$ dos pacientes tratados com placebo. No geral, os pacientes tratados com antidepressivos relataram uma taxa mais alta de efeitos adversos do que os pacientes tratados com diazepam (Rickels et al., 2010).

Estudos experimentais demonstraram a atividade ansiolítica do óleo essencial de Angelica sinensis. Em um deles foi comparado com o ansiolítico diazepam utilizando os testes comportamentais da caixa claro-escuro e também o teste de hipertermia induzida por estresse, que demonstrou que 6o e 70 minutos após a administração do medicamento, o óleo essencial de angélica na dose de $30,0 \mathrm{mg} / \mathrm{kg}$ inibiu a hipertermia induzida por estresse e em outro estudo, houve efeito ansiolítico demonstrado pela melhora na interação social e redução do comportamento agressivo de roedores (Chen et al., 2004; Min et al., 2005).

\subsection{Aspectos relacionados à fisiopatologia e tratamentos de transtornos depressivos}

Entre os transtornos emocionais, a depressão se configura como uma das patologias mais incapacitantes do mundo. $\mathrm{Na}$ maioria das vezes o transtorno depressivo se desenvolve concomitantemente com a ansiedade patológica. Alguns autores defendem que o agravamento da ansiedade pode estar relacionado com a depressão, de forma que o tratamento dos distúrbios ansiosos podem ser uma boa alternativa para a prevenção dos transtornos depressivos (Ribeiro et al., 2010).

Estudos sobre a eficácia da Homeopatia na depressão são escassos e poucos relatos de evidências clínicas de casos são encontrados. Até mesmo nos estudos sobre fármacos ansiolíticos convencionais, muitos pesquisadores questionam sobre a eficácia dos medicamentos alegando diferenças entre os modelos comportamentais empregados nas pesquisas. Um estudo evidenciou diferenças significativas nos efeitos de medicamentos agonistas serotonérgicos, considerados efetivos para o tratamento da depressão, entre o teste de Labirinto em Cruz Elevado e Teste de 
Emergência, ambos considerados modelos de depressão, levantando dúvidas sobre os efeitos encontrados (Lalonde \& Strazielle, 2010).

Um estudo relatou a evolução de um tratamento para depressão em is pacientes do SUS da cidade de Jundiaí, São Paulo, Brasil, em 2006 no qual foi encontrada redução da depressão em I4 dos pacientes tratados após 7 semanas, sugerindo que a Homeopatia pode ser uma alternativa terapêutica no tratamento da depressão, contudo, os autores deixam claro que estudos randomizados e controlados são necessários sobre a eficácia e segurança do tratamento (Adler et al., 2013).

Muitos são os transtornos que se apresentam associados à depressão ou ansiedade, o que aumenta a complexidade do tratamento. $\mathrm{O}$ isolamento social é um sintoma comum à ambos os transtornos de humor: depressão e Transtorno de Ansiedade Social (TAS), bem como as preocupações recorrentes do TAS podem ocorrer em outros transtornos psiquiátricos, como o transtorno da ansiedade generalizada. Desta forma, o tratamento farmacológico disponível na alopatia pode ser comum a ambos os transtornos de humor (Lader et al., 2004).

A ocorrência de efeitos adversos pelo uso de medicamentos prejudica a qualidade de vida dos pacientes. Um estudo randomizado, controlado por placebo, de dose fixa e referência ativa, incluindo 47 centros em ir países verificou que o medicamento escitalopram, da classe dos inibidores de recaptação de serotonina é eficaz no tratamento do TAS, comparado ao fármaco de referência Paroxetina, que é classicamente recomendado na terapêutica da depressão. Contudo, o estudo relatou grande ocorrência de náuseas nos pacientes (Levitan et al., 20II).

Além disto, é discutido o risco de suicídio associado ao uso de antidepressivos. Um estudo de coorte de 12 meses de observação em 2,4 milhões de pacientes com depressão dos Estados Unidos entre de 1999 a 2006 comparou o risco de suicídio com o uso de antidepressivos e relatou que a descontinuação do antidepressivo teve um risco significativo de tentativa de suicídio, assim como quando o período terapêutico era abreviado. Segundo este estudo, o risco de suicídio também possui associação aos períodos de mudanças de dose (Valuck et al., 2009).

Muitos estudos descreveram síndromes de descontinuação dos antidepressivos, como os da classe dos Inibidores de Recaptação de Serotonina (ISRS) e antidepressivos Tricíclicos (TCAs), que provocam sintomas como: tontura, náusea/vômito, dor de cabeça e letargia, enquanto os da classe dos Inibidores da enzima Mono Amino Oxidase (IMAO) levam à ocorrência de sintomas mais graves, com comprometimentos cognitivos e delírios, psicose, depressão, distúrbios do pensamento, mania, agitação, agressividade e insônia (Tamam \& Ozpoyraz, 2002). 
Paralelamente aos estudos clínicos, trabalhos experimentais com animais de laboratório demonstraram que a buspirona, administrada em baixas concentrações altera atividade motora dos animais testados em campo aberto, aumentando a latência para se mover, Além disto, o fármaco promove alterações neuroquímicas, diminuindo o metabolismo de dopamina na região do estriado (Shireen \& Haleem, 2005).

Zulian Teixeira afirma que a suspensão de medicamentos psiquiátricos ansiolíticos e antidepressivos pode desencadear agravação rebote do quadro inicial. Segundo ele, o reaparecimento da doença de base ocorre na ausência do efeito farmacológico do medicamento, o que no medicamento homeopático ultra diluído não ocorre, posto que o princípio de cura é pelo semelhante (Zulian, 2013).

Considerando os efeitos prejudiciais decorrentes do tratamento dos transtornos de ansiedade e depressão, pelo uso de medicamentos convencionais, o uso da Homeopatia pode ser a chave para o tratamento da ansiedade e depressão de forma a anular o efeito rebote destes fármacos (Grimaldi-Bensouda et al, 2016; Chirumbolo, 2015).

\subsection{Considerações sobre o medo e tratamentos complementares}

O medo é uma emoção fundamental, presente em todas as raças, espécies, culturas e idades. Em relação aos padrões de resposta de medo, a evitação ou fuga, a imobilidade, a agressividade ou a submissão podem ser elicitadas perante um estímulo ameaçador. O medo pode ser diferenciado da ansiedade de acordo com a presença ou ausência do evento desencadeador (Zamberlam et al, 2016).

A presença iminente da ameaça define o medo e a sua ausência caracteriza a ansiedade. Ainda, consideram-se os comportamentos de fuga ou evitação característicos dos dois estados, onde no medo o comportamento de fuga torna-se característico, enquanto na ansiedade não ocorre evitação, posto que não há estímulo ameaçador, sendo assim modulada pelo estado emocional (Baptista et al, 2005).

Tetau e Tetau deram início em 1960 aos primeiros testes em psicofarmacologia utilizando medicamentos homeopáticos. Eles mostraram que doses Thuya na diluição de ${ }_{9} \mathrm{CH}$ restabeleceram o equilíbrio psíquico de ratos, restabelecendo seu condicionamento normal (Tétau, 2000).

Existem controvérsias em relação aos estudos de comportamento em animais, quando se trata de Homeopatia, considerando a individualidade do tratamento. Os testes comportamentais em animais, quando realizados de maneira isolada, podem não refletir uma imagem clara $e$ 
abrangente do perfil emocional de um animal. Portanto, muitos autores testam as suas hipóteses experimentais usando uma série de testes relacionados pensados para refletir diferentes facetas da emocionalidade. Estudos clínicos são necessários para esclarecer os efeitos dos medicamentos dinamizados nos seres humanos (Bellavite et al., 2009).

Bellavite e colaboradores demonstraram em 2012 os efeitos significativos de Gelsemium em diferentes potências na modulação da resposta emocional de camundongos avaliados em diferentes testes comportamentais e com variações metodológicas nos modelos de campo aberto e na caixa claro-escuro (Bellavite et al., 2012).

Alguns testes comportamentais analisam a memória do medo condicionado, que representa uma forma de avaliar a aquisição do medo pelo método do condicionamento clássico. Este experimento foi delineado em 1927 por Ivan Pavlov em cães, onde tocava um sino (o estímulo condicionado; CS), imediatamente antes de dar o seu alimento de cães (carne, o estímulo não condicionado), que induzia a salivação nos cães (a resposta incondicionada; IR). Após repetidas apresentações de sino e carne (CS-IR), os cães de Pavlov salivavam ao som do sino, mesmo sem a apresentação da carne, gerando a resposta condicionada (CR) (Zamberlam et al., 2016; Filho et al., 2010).

O condicionamento Pavloviano, como foi denominado, até hoje é um modelo amplamente utilizado para o estudo das bases neurobiológicas da memória associativa (Filho et al., 2010).

Um extrato seco padronizado de Ginkgo biloba (EGb), que contém 24\% de glicosídeos ginkgo flavonoides e 6\% terpenoides, é utilizado na elaboração de medicamentos fitoterápicos. Diferentes propriedades terapêuticas são descritas para o EGb, como eficazes para a memória, problemas de concentração, confusão, depressão, ansiedade, tonturas, zumbidos e dores de cabeça (Yang et al., 2009; yang et al., 2007).

Estudos apontam que o Ginkgo biloba pode ser útil no tratamento de transtornos de ansiedade e medo. A administração sistêmica do extrato EGb facilitou a extinção do medo condicionado de maneira dependente da dose. Além disto, pesquisadores evidenciaram que os efeitos fisiológicos encontrados estão baseados na regulação de neurotransmissores (Zamberlam et al., 2016, 2017).

Pesquisas recentes em tarefas de supressão condicionada do comportamento de lamber, em ratos, mostraram que diferentes doses de extrato padronizado de Ginkgo biloba promovem diferentes efeitos comportamentais em ratos, sendo que a menor dose testada, $240 \mathrm{mg} / \mathrm{kg}$ facilitou a aquisição da memória do medo condicionado, já a dose intermediária, $500 \mathrm{mg} / \mathrm{Kg}$ 
revelou efeitos ansiolíticos significativos e a maior dose, $1000 \mathrm{mg} / \mathrm{Kg}$ apresentou efeito melhorador cognitivo para a retenção da memória aversiva (Zamberlam et al, 2016).

Um estudo mostrou que Ignatia amara foi capaz de modular as respostas emocionais em camundongos no paradigma da caixa claro-escuro e possivelmente possui algum efeito no comportamento de urinar dos animais (Marzotto et al., 20II).

De acordo com a Matéria Médica Homeopática, Ignatia amara tem uma ação marcante em várias condições mentais, tais como tristeza, ansiedade, angústia, depressão, desapontamento, melancolia, os quais podem ser causados após receber más notícias ou perdas afetivas e supressão do comportamento sexual (Lathoud, 2017).

Para Gelsemium os sintomas relatados nos compêndios homeopáticos tais como medo de morrer, medo que o coração pare, medo de se mover, tendência de isolamento (estar só), intensa prostração com tremores e ausência de sede, também fazem parte do quadro de sintomas encontrados em transtornos de ansiedade, depressão e medo. Gelsemium é tradicionalmente usado devido aos seus efeitos ansiolíticos evidenciados em modelos animais pelo seu extrato ou por diluições homeopáticas (Hahnemann, 20or; Bellavite et al., 2017).

Não se encontram na literatura estudos com medicamentos dinamizados derivados de Ginkgo biloba. Além disto, o uso de medicamentos homeopáticos em modelos de comportamento que avaliem o medo ainda carece de estudos. Estes estudos baseados em fitoterapia fornecem fortes justificativas para o uso destas substâncias em medicamentos homeopáticos em baixas potências.

As terapias atuais direcionadas para a ansiedade envolvem o alvo farmacológico ácido gama-aminobutírico (GABA), que está relacionado com o mecanismo inibitório no sistema nervoso central, mas podem causar efeitos indesejáveis e prejuízo na memória e sintomas de abstinência.

Uma revisão sistemática evidenciou várias plantas que possuem interação com o neurotransmissor GABA, tais como kava-kava, valeriana, camomila, Ginkgo biloba, maracujá, erva-cidreira, entre outras. Entre os estudos encontrados figuram investigações pré-clínicas e ensaios em seres humanos, revelando que há vários fitomedicamentos que modulam a via GABAérgica, classicamente envolvida no controle da ansiedade e com a vantagem de apresentar segurança em relação aos efeitos adversos e colaterais (De Oliveira et al., 2017).

Savage, em 2018 realizou uma revisão sistemática de Io anos sobre evidências de ensaios clínicos com fitoterápicos e encontrou evidências para Piper methysticum (Kava), Passiflora spp. e Galphimia glauca na ansiedade e Hypericum perforatum, Crocus sativus (açafrão) na depressão e 
Ginkgo biloba (Ginkgo) na esquizofrenia. Contudo, foi observado diminuição nos projetos de pesquisa envolvendo estudos com fitoterápicos (Savage et al, 2018).

Tendo em vista os grandes prejuízos no desempenho laboral e na qualidade de vida dos indivíduos acometidos com transtornos de ansiedade e que estes efeitos podem se tornar crônicos interferindo na cognição e memória, dificuldade de atenção, bem como sintomas fisiológicos como insônia e tensão, faz-se necessário a busca de terapias complementares que auxiliem neste quadro.

\section{DISCUSSÃO}

Estudos baseados em fitoterapia fornecem evidências sobre os efeitos de extratos de plantas em transtornos emocionais, como a ansiedade. Por este motivo justifia-se o uso destas substâncias em baixas potências em medicamentos dinamizados homeopáticos. Foi visto que um extrato padronizado de Ginkgo biloba (EGb) reduziu a taxa de medo dos animais após o tratamento com uma dose intermediária, mas não em altas ou baixas doses, refletindo que o efeito encontrado não necessariamente é dependente da dose (Zamberlam et al., 20ı6; Zamberlam \& Cerutti, 2017).

Este mesmo grupo de pesquisa avaliou a resposta de animais de laboratório no comportamento de extinguir o medo aversivo, mediante o tratamento com frações enriquecidas de flavonoides de uma planta brasileira denominada Erythrina falcata, onde os resultados evidenciaram uma significativa redução da extinção do medo condicionado, sugerindo que os flavonoides presentes nesta planta podem auxiliar em terapias cognitivo-comportamentais para a extinção do medo (De Oliveira et al., 2017).

Sabe-se que a ansiedade pode ser decorrente de um estresse intenso, comum na vida cotidiana atual, não sendo considerado um evento negativo e pode ser motivador e estimulante. Contudo, quando o estresse se apresenta em intensidade excedente à capacidade de adaptação do indivíduo, pode ocasionar desequilíbrio no circuito neural que comanda as esferas cognitivas, memória, humor e alterando funções fisiológicas como a liberação de hormônios e neurotransmissores (Kurebayashi et al., 2016).

O uso da medicina complementar e alternativa na ansiedade resulta em redução dos efeitos colaterais dos medicamentos ansiolíticos clássicos. Um decocto de ervas denominado Gan-Mai-Da-Zao (GMDZ), preparado com Triticum, Glycyrrhiza e Zizyphi Fructus, se mostrou eficiente no tratamento de doenças emocionais e estes efeitos parecem envolver os neurotransmissores serotonina e GABA (Chen et al., 2019). 
Estudos em animais analisaram o uso do decocto GMDZ em modelos animais de distúrbios neuropsiquiátricos e evidenciaram melhora nos níveis de estresse psicológico agudo, estresse pós-traumáticos e depressão leve. Entretanto, uma revisão sistemática com meta análise analisou ensaios clínicos e randomizados do uso da decocção GMDZ no tratamento da depressão e forneceu evidências de que a mistura chinesa não é eficiente para tratamento da depressão maior, pós-cirúrgica ou em idosos ( $\mathrm{Su}$ RK et al., 2017; jun. et al., 2014).

Alguns autores têm questionado a qualidade dos estudos clínicos randomizados em Homeopatia e apontam efeitos restritos e não significativos decorrentes do tratamento homeopático individualizado. Além disto, os estudos farmacológicos que explicam os mecanismos de ação dos medicamentos ultra diluídos ainda são carentes (Mathie, 2015).

O desenvolvimento de novos alvos terapêuticos a partir de plantas medicinais ou fontes minerais é requerido para redução dos efeitos colaterais promovidos por medicamentos ansiolíticos, antidepressivos e para o controle das emoções de medo. Um estudo avaliou os efeitos ansiolíticos de extratos de raízes de Gelsemium sempervirens em modelo animal de labirinto em cruz elevado, classicamente utilizado para medir ansiedade. Foi utilizado o medicamento Diazepam controle positivo e como resultados foi visto que o extrato metanólico de Gelsemium claramente melhorou os comportamentos de ansiedade dos animais (Dutt et al., 2010).

Grupos de pesquisa se dedicam a investigar os mecanismos de ação de Gelsemium sempervirens em modelos in vivo e in vitro e os resultados sugerem efeito no sistema nervoso central em doses muito baixas e em diluições homeopáticas. Embora os estudos não tenham sido refutados, levantaram questionamentos sobre os métodos de investigação (Magnani et al., 2010).

\section{CONCLUSÃO}

Pesquisas evidenciaram que o tratamento homeopático resultou em maior taxa de redução da ansiedade, comparado com os ansiolíticos convencionais, além de não resultar em efeitos colaterais, o que ocorre com os medicamentos alopáticos tradicionalmente usados para o controle da ansiedade.

Entretanto, os efeitos ansiolíticos ou de controle de sintomas de ansiedade, decorrentes do tratamento com Homeopatia, encontrados em artigos publicados em revistas indexadas, ainda não são representativos, sendo necessários estudos mais robustos, clínicos, randomizados de modo a fortalecer o corpo de evidências da eficácia da Homeopatia no restabelecimento da saúde. 
É consenso que a Homeopatia é uma opção de terapêutica promissora no sentido de buscar o equilíbrio do paciente por estimular reação semelhante à doença em indivíduo sadio, em busca da cura, ao contrário do efeito paliativo observado nas drogas convencionais disponíveis.

\section{REFERÊNCIAS}

I. ADLER UC, KRÜGER S, TEUT M, LÜDTKE R, SCHÜTZLER L, MARTINS F, WILLICH SN, LINDE K, WITT CM. Homeopathy for depression: a randomized, partially double-blind, placebo-controlled, four-armed study (DEP-HOM). PLoS One. 2013. 8(9):e74537. doi: I0.1371/journal.pone.0074537. PMID: 24086352; PMCID: $\mathrm{PMC}_{3781106 .}$

2. ANDREATINI R, BOERNGEN-LACERDA R, ZORZETTO FILHO D. Tratamento farmacológico do transtorno de ansiedade generalizada: perspectivas futuras. Rev Bras Psiquiatr 2001; 23(4):233-42.

3. ARAÚJO LFSC. DALGALARRONDO P, BANZATO CEM. Sobre a noção de causalidade na medicina: aproximando Austin Bradford Hill e John L. Mackie. Rev Psiq Clín. 2014;4I(2):56-6r.

4. BAPTISTA A, CARVALHO M, LORY F. O medo, a ansiedade e as suas perturbações. Psicologia. 2005. $19(\mathrm{I}-2), 267-277.2$ Disponível em: $<$ http://www.scielo.mec.pt/scielo.php?script=sci_arttext\&pid=So8742049200500o10oor3\&lng=pt\&nrm=iso>. ISSN 0874-2049. Acessado em i6/ri/ 2019.

5. BARETTA IP, FELIZARDO RA, BIMBATO VF, SANTOS MGJ, KASSUYA CAL, GASPAROTTO JUNIOR A, SILVA CR, OLIVEIRA SM, FERREIRA J, ANDREATINI R. Anxiolytic-like effects of acute and chronic treatment with Achillea millefolium L. extract. Journal of Ethnopharmacology. 2012; I4O(I): 46-54, doi:Io.Ior6/j.jep.2011.II.047.

6. BATELAAN NM, RHEBERGEN D, SPINHOVEN P, VAN BALKOM AJ, PENNINX BW. Two-year course trajectories of anxiety disorders: Do DSM classifications matter?. J. Clin Psychiatry[Internet]. 2014; 75(9):985 - 93. Disponível em: https://www.psychiatrist.com/jcp/article/pages/2014/v75no9/v75nogr8.aspx. Acessado em I4/II/2019. 
7. BELLAVITE P, BONAFINI C, MARZOTTO M. Experimental neuropharmacology of Gelsemium sempervirens: Recent advances and debated issues. Journal of Ayurveda and Integrative Medicine. 2018;9(I):69 - 74. ISSN 0975-9476. doi.org/ro.1016/j.jaim.2017.or.oro.

8. BellaVite P, CONFORTI A, MARZOTTO $\mathrm{M}$, et al. Testing Homeopathy in Mouse Emotional Response Models: Pooled Data Analysis of Two Series of Studies. Evidence-Based Complementary and Alternative Medicine. 2012. 9p. Article ID 954374. https://doi.org/10.1155/2012/954374.

9. BELlAVITE P, MAGNANI P, MARZOTTO M, CONFORTI A. Assays of homeopathic remedies in rodent behavioural and psychopathological models. Homeopathy 2009; 98(04): 208-227. doi:10.1016/j.homp.2009.09.005.

Io. BOUSTA R, SOULIMANI I, JARMOUNI P, BELON J, FALLA N, FROMENT C, YOUNOS. Neurotropic, immunological and gastric effects of low doses of Atropa belladonna L., Gelsemium sempervirens L. and Poumon histamine in stressed mice. Journal of Ethnopharmacology. 2001; 74(3): 205-215. ISSN 0378-8741. doi.org/ro.1016/So3788741(oo)oo346-9.

II. BRASIL MINISTÉRIO DA SAÚDE. 2006. Série B. Textos Básicos de Saúde. 92 p. ISBN 85-334-I208-8. Disponível em: http://bvsms.saude.gov.br/bvs/publicacoes/pnpic.pdf. Acessado em io/ıI/2o19.

12. CASTILlO ARGL, RECONDO R, ASBAHR FR, MANFRO GG. Transtornos de ansiedade. Brazilian Journal of Psychiatry. 2000. 22(Suppl. 2), 20-23. doi:10.1590/Si51644462000000600006 .

13. CERVO L. \& TORRI, V. Psychopharmacology 220: 439. 2012. Online ISSN 1432-2072. https://doi.org/10.1007/soo213-011-2582-z. II.

14. CHEN HS, GU LJ, YANG YX, GUO JY. GABA and 5-HT Systems Are Involved in the Anxiolytic Effect of Gan-Mai-Da-Zao Decoction. Frontiers in Neuroscience. 2019; (12). DOI =10.3389/fnins.2018.01043. ISSN=1662-453X.

15. CHEN SW, MIN L, LI WJ, KONG WX, LI JF, ZHANG YJ. The effects of angelica essential oil in three murine tests of anxiety. Pharmacology Biochemistry and Behavior. 2004. 79 (2): $377-382$. 
16. CHIRUMBOLO S. On Gelsemium and Complementary and Alternative Medicine (CAM) in Anxiety and Experimental Neurology. Neurol Ther. 2015;4(I):I-10. doi:10.1007/s40120-014-0025-6.

17. COLIN P. An epidemiological study of a homeopathic practice. The British Homeopathic Journal. 2000; 89(3):116-21.

18. CORREAA, AD; QUINTAS, L. E. M.; SIQUEIRA-BATISTA, R. Similia Similibus Curentur: revisitando aspectos históricos da homeopatia nove anos depois. História, Ciências, Saúde. Manguinhos. 2006; 13(I):13-3I.

19. DE OLIVEIRA DR, ZAMBERLAM CR, GAIARDO RB, RÊGO GM, CERUTTI JM, CERUTTI SM. Flavones from Erythrina falcata are modulators of fear memory. 2014. BMC Complement Altern Med I4: 288.

20. DE OLIVEIRA, DR, ZAMBERLAM, CR, RÊGO, GM, CAVALHEIRO A, CERUTTI JM, CERUTTI SM. Effects of a Flavonoid-Rich Fraction on the Acquisition and Extinction of Fear Memory: Pharmacological and Molecular Approaches. Frontiers in Behavioral Neuroscience. 2016;345(9):1-21). doi:10.3389/fnbeh.2015.00345.

2I. DUTT V, DHAR VJ, SHARMA A. Antianxiety activity of Gelsemium sempervirens. Pharmaceutical Biology. 2010;48(10):1091-1096. DOI: 10.3109/13880200903490521.

22. ElEUTÉRIO ASL, DE OLIVEIRAL DSB, PEREIRA JÚNIOR ES. Homeopatia no controle do medo e ansiedade ao tratamento odontológico infantil: Revisão. Rev. Odontol. Univ. Cid. São Paulo 2011; 23(3): 238- 44. ISSN 1983-5183.

23. ENNACEUR A, MICHALIKOVA S, VAN RENSBURG R, CHAZOT PL. Tolerance, sensitization and dependence to diazepam in Balb/c mice exposed to a novel open space anxiety test. Behavioural Brain Research. 2010. 209(I): 154-164. doi:10.1016/j.bbr.2010.01.039.

24. FERNANDES MA, RIBEIRO HKP, SANTOS JDM, MONTEIRO CFS, COSTA RS, SOARES RFS. Prevalence of anxiety disorders as a cause of workers' absence. Rev Bras Enferm 2018;71(Suppl 5):2213-20. [Thematic Issue: Mental health] doi: http://dx.doi.org/10.1590/o034-7167-2017-0953. 
25. FILHO AC, FAKOURY MK, FERRY FRA. Ginkgo biloba e memória - revisão sistemática. Rev. Bras. Geriatr. Gerontol., Rio de Janeiro, 2010; I3(I):I45-152.

26. FONTES OL. Manole. 2013. ISBN:978-85-204-3646-2.

27. GIORGI MS, NETO LB, FRIAS, AC, SANTOS CMS; TRINDADE. Contribuição da homeopatia no controle da ansiedade e do medo, como prevenção das emergências médicas em odontologia: estudo piloto. Revista de Homeopatia. 2010;73(3/4): 17-22. [10].

28. GRAEFF FG. Aspectos neuroquímicos: o papel da serotonina no transtorno obsessivocompulsivo. Rev Bras Psiquiatr. 200I. 23 (supl. 2): 35-37.

29. GRAEFF, FG. Psychopharmacology (2002) I63: 467. doi: 10.1007/soo213-002-III2-4.

30. GREENWOOD DJ. Medicina intervencionista vs. Medicina naturalista: história antropológica de uma pugna ideológica. Arxiu d'Etnografia de Catalunya. 2016; (3): 588I.p.I4.

31. GRIMALDI-BENSOUDA L, ABENHAIM L, MASSOL J, et al. Homeopathic medical practice for anxiety and depression in primary care: the EPI3 cohort study. BMC Complement Altern Med. 2016; I6:125. doi:Io.II86/si29o6-oi6-IIO4-2.

32. GRUNDMANN O, NAKAJIMA J, SEO S, BUTTERWECK V. Anti-anxiety effects of Apocynum venetum L. in the elevated plus maze test. Journal of Ethnopharmacology. 2007;110(3):406-II. doi:10.1016/j.jep.2006.09.035.

33. HAHNEMANN S. Organon da arte de curar. 6. ed. São Paulo: Editora Robe Editorial, 200I. 248p.

34. HORVilleUR, AlAiN. São Paulo; Vade Mecum da Prescrição em Homeopatia. Organização Andrei Ed; 2003. 653 p.

35. JUN JH, TAE-YOUNG C, JU AL, KYUNG-JIN Y, MYEONG SL. Herbal medicine (Gan Mai Da Zao decoction) for depression: A systematic review and meta-analysis of randomized controlled trials. Maturitas, 2014;4(79):370-380. DOI: https://doi.org/10.1016/j.maturitas.2014.08.oo8.

36. KUREBAYASHI LFS, TURRINI RNT, KUBA G, SHIMIZU MHM, TAKIGUCHI RS. Chinese phytotherapy to reduce stress, anxiety and improve quality of life: 
randomized controlled trial. Rev Esc Enferm USP. 2016;50(5):853-86o. DOI: http://dx.doi.org/10.1590/Soo8o-6234201600oo60oo20

37. LADER M, STENDER K, BÜRGER V, NIL R. Efficacy and tolerability of escitalopram in 12- and 24-week treatment of social anxiety disorder: Randomised, double-blind, placebo-controlled, fixed-dose study. Depression and Anxiety. 2004. 19(4), 241-248. doi:10.1002/da.20014.

38. LALONDE R, STRAZIELLE C. Relations between open- field, elevated plus-maze, and emergence tests in $\mathrm{C}_{57} \mathrm{BL} / 6 \mathrm{~J}$ and $\mathrm{BALB} / \mathrm{c}$ mice injected with $\mathrm{GABA}$ - and $5 \mathrm{HT}$ anxiolytic agents. Fundamental and Clinical Pharmacology. 2010. 24(3); 365-376. doi.org/10.1111/j.1472-8.

39. LATHOUD, J.A. Estudos de Matéria Médica Homeopática, tradução de Heloísa Helena

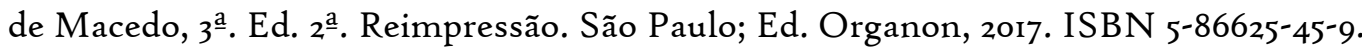

40. LEVITAN MN, CHAGAS MHN, CRIPPA JAS, MANFRO GG, HETEM LAB, ANDRADA NATHAliA C. et al. Diretrizes da Associação Médica Brasileira para o tratamento do transtorno de ansiedade social. Rev. Bras. Psiquiatr. 2011; 33(3): 292-302. doi:10.1590/S1516-44462011000300o14.

41. LIU M, HUANG HH, YANG J, SU YP, LIN HW, LIN LQ, LIAO WJ, YU CX. The active alkaloids of Gelsemium elegans Benth. are potent anxiolytics. Psychopharmacology (Berl). 2013;225(4):839-51.

42. MAGNANI P, CONFORTI A, ZANOLIN E, MARZOTTO M, BELLAVITE P. Dose-effect study of Gelsemium sempervirens in high dilutions on anxiety-related responses in mice. Psychopharmacology (Berl). 2010; 210(4):533-545. doi:10.1007/soo213o10-1855-2.

43. MAMTANI R \& CIMINO A. A primer of complementary and alternative medicine and its relevance in the treatment of mental health problems. 2002. Psychiatr Q 73:367381.

44. MARZOTTO M, CONFORTI A, MAGNANI P, ZANOLIN ME, BELLAVITE P. Effects of Ignatia amara in mouse behavioural models. Homeopathy. 2012.10I(I): 57-67. doi:10.1016/j.homp.2011.10.oor. 
45. MATHIE RT. Controlled clinical studies of homeopathy. Homeopathy 2015; 104(04): 328-332

DOI: 10.1016/j.homp.2015.05.003.

46. MAX TÉTAU. Matéria Médica Homeopática Alvos Específicos. Organização Andrei Editora Ltda. São Paulo, SP. 20oo. Pag. 57 - 58.

47. MENEZES AKS, MOURA LF, MAFRA VR. Transtorno de ansiedade generalizada: uma revisão da literatura e dados epidemiológicos. Revista Amazônia Science \& Health. 2017; 5(3):42-49. doi:10.18606/2318-1419/amazonia.sci.health.

48. MIN L, CHEN SW, LI WJ, WANG R, LI YL, WANG WJ, MI XJ. The effects of angelica essential oil in social interaction and hole-board tests. Pharmacology Biochemistry and Behavior. 2005. 8I(4), 838-842. doi:10.1016/j.pbb.2005.05.015.

49. MORAES CF, SILVA NP. Saúde mental $e$ as relações de trabalho: como a ansiedade influencia o comportamento humano no ambiente de trabalho. Interfaces Saberes. 2015; I4(I):I-I6. Disponível em: https://interfacesdesaberes.faficape.edu.br/index.php/importI/article/view/533/274. Acessado em I5/ri/2019.

5o. ORGANIZAÇÃO MUNDIAL DE SAÚDE-OMS. Depression and other common mental disorders: global health estimates. Geneva: WHO; 2017. Disponível em: http://apps.who.int/iris/bitstream/10665/254610/r/WHO-MSD-MER-2017.2-eng.pdf. Acessado em I2/II/2019.

51. PUSTIGLIONE M, GOLDENSTEIN E, CHENCINSKI MY. Homeopatia: um breve panorama desta especialidade médica. Revista de Homeopatia. 2017a; 8o(I/2):I-17.

52. PUSTIGLIONE M. A Homeopatia e o romantismo brasileiro, 2017. Disponível em: marcelopustiglione.com. Acessado em Io/II/2019.

53. PUSTIGLIONE M. A homeopatia na literatura. Monteiro Lobato um arauto da Homeopatia no período pré-modernista brasileiro, 2017b. Disponível em marcelopustiglione.com.

54. RAVINDRAN LN, STEIN MB. The pharmacologic treatment of anxiety disorders: a review of progress. J Clin Psychiatry. 2010. 7I(7): 839-854.206.2009.00772.x. 
55. ROBINSON T. Responses to homeopathic treatment in National Service general practice. Homeopathy. 2006; 95:9-I4.

56. SÁNCHEZ LIÉVANO CV. Breve mirada al desarrollo de la historia de la homeopatia em el mundo durante los dos últimos siglos. 2013. (Doctoral dissertation, Universidad Nacional de Colombia).

57. SARRIS J. Herbal medicines in the treatment of psychiatric disorders: ro-year updated review. Phytotherapy Research. 2018; 32: 1147-II62. https://doi.org/10.1002/ptr.6055.

58. SAVAGE K, FIRTH J, STOUGH C, SARRIS J. GABA-modulating phytomedicines for anxiety: A systematic review of preclinical and clinical evidence. Phytotherapy Research. 2018; 32: 3-18. https://doi.org/10.1002/ptr.5940.

59. SCHMIDT DRC, DANTAS RAS, MARZIALE MHP. Ansiedade e depressão entre profissionais de enfermagem que atuam em blocos cirúrgicos. Rev. Esc. Enferm. USP [online]. 2011. 45 (2):487-493.

6o. SHARPLES FMC, VAN HASELEN R, FISHER P. NHS patients' perspective on complementary medicine: a survey. Complement Ther Medicine. 2003; II:243-8.

61. SHIREEN E, HALEEM DJ. Motor effects of buspirone: relationship with dopamine and serotonin in the striatum. Journal of College of Physicians and Surgeons Pakistan. 2005. I5;(12); 753-756.

62. SU RK, HYE WL, JUN JH, BYOUNG-SEOB K. Effects of Herbal Medicine (Gan Mai Da Zao Decoction) on Several Types of Neuropsychiatric Disorders in an Animal Model: A Systematic Review: Herbal medicine for animal studies of neuropsychiatric diseases. J Pharmacopuncture. 2017;20(I):5-9. doi: 10.3831/KPI.2017.20.005.

63. TAMAM L, OZPOYRAZ N. Selective serotonin reuptake inhibitor discontinuation syndrome: A review. 2002. Adv Therapy. 19(I):17-26. doi.org/10.1007/BFo2850015.

64. TEIXEIRA MA. La evidencia científica del modelo epistemológico homeopático. La Homeopatía de México. 2012; 82(685): 5-20. 
65. VALUCK RJ, ORTON HD, LIBBY AM. Antidepressant discontinuation and risk of suicide attempt: a retrospective, nested case-control study. J Clin Psychiatry. 2009; 70:1069-77.

66. YANG YL, HSIEH CW, WO YY, YANG YC, LU KT. Intra-amygdaloid infusion of Ginkgo biloba leaf extract (EGb76r) facilitates fear-potentiated startle in rats. Psychopharmacology.2009.202(I-3):187-96. doi: 10.1007/s00213-008-II38-3.

67. YANG YL, SU YW, NG MC, CHAO PK, TUNG LC, LU KT. Extract of Ginkgo biloba EGb76r Facilitates Extinction of Conditioned Fear Measured by Fear-Potentiated Startle. Neuropsychopharmacology.2007.32(2):332-42.

68. ZACHARIAS, C.R. (2002). Physical research in dynamized systems. Medical Hypotheses, 58(6), 523-526. doi:10.1054/mehy.2001.1508.

69. ZAMBERLAM CR, CERUTTI, SM. Protective, Preventive and Enhancing-Memory Effects of the Flavonoidic-Rich Plants or Flavonoidic Molecules: Behavioural, Neurochemical and Pharmacological Correlates. International Journal of Complementary and Alternative Medicine.2017;6(5):00200.

DOI: 10.15406/ijcam.2017.06.00200.

7o. ZAMBERLAM CR, VENDRASCO NC, OLIVEIRA DR, GAIARDO RB, CERUTTI SM. Effects of standardized Ginkgo biloba extract on the acquisition, retrieval and extinction of conditioned suppression: Evidence that short-term memory and long-term memory are differentially modulated. Physiol Behav. Elsevier B.V.; 2016; 165:55-68.

71. TEIXEIRA, M.Z. (2013). Efeito rebote dos fármacos modernos: evento adverso grave desconhecido pelos profissionais da saúde. Revista da Associação Médica Brasileira, 59(6), 629-638. https://doi.org/ro.1016/j.ramb.2013.05.003 